\title{
Simultaneous finding of cleistothecia, Hülle cells, conidial heads, and calcium oxalate crystals in a fungal ball within the maxillary sinus, alongside dental root canal-filling material
}

\author{
Masatomo Kimura Pathology, Kindai Univ Faculty of Medicine Osaka-Sayama, Japan
}

Objective

The present case illustrates a very rare combination of cleistothecia, Hülle cells, conidial heads, and calcium oxalate crystals in addition to dental root canal-filling material in a fungal ball of the human maxillary sinus.

Method

Histology of the specimen removed from the right maxillary sinus of a 51-year-old man was studied. Hematoxylin and eosin stain was performed, and the histology sections were observed under regular or polarized light. A scanning electron microscope (SU3500; Hitachi, Tokyo, Japan) equipped with an energy-dispersive X-ray analyzer (Genesis APEX; Ametek, PA, USA) was used to do element analysis of a foreign body in the section.

Conclusion

The combination of cleistothecia and Hülle cells is suggestive of Aspergillus nidulans, while the brown conidia with calcium oxalate crystals are indicative of Aspergillus niger. Therefore, simultaneous proliferation of the two different fungi is likely in the present fungal ball. Gutta-percha point, a dental root canal-filling material, was found in the center of the fungal ball, suggesting that it served as a core.

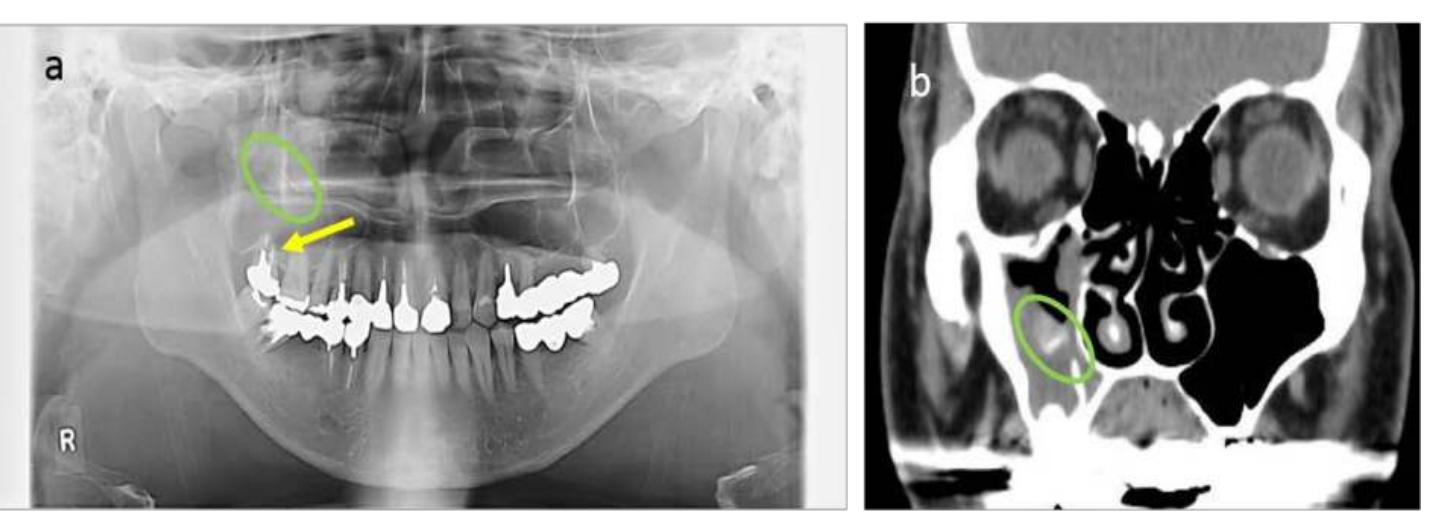

Fig. 1 (a) Dental panoramic x-ray film. Filling material is seen in the root canals of multiple teeth, including the upper right second molar (arrow). Needle-shaped material (encircled), which shows similar opacity to that of the root-filling material, is seen in the maxillary sinus. (b) Pre-operative coronal plain computed tomography (CT) of the face. Soft tissue-dense opacity with metal-dense needle-shaped material (encircled) is seen occupying the right maxillary sinus
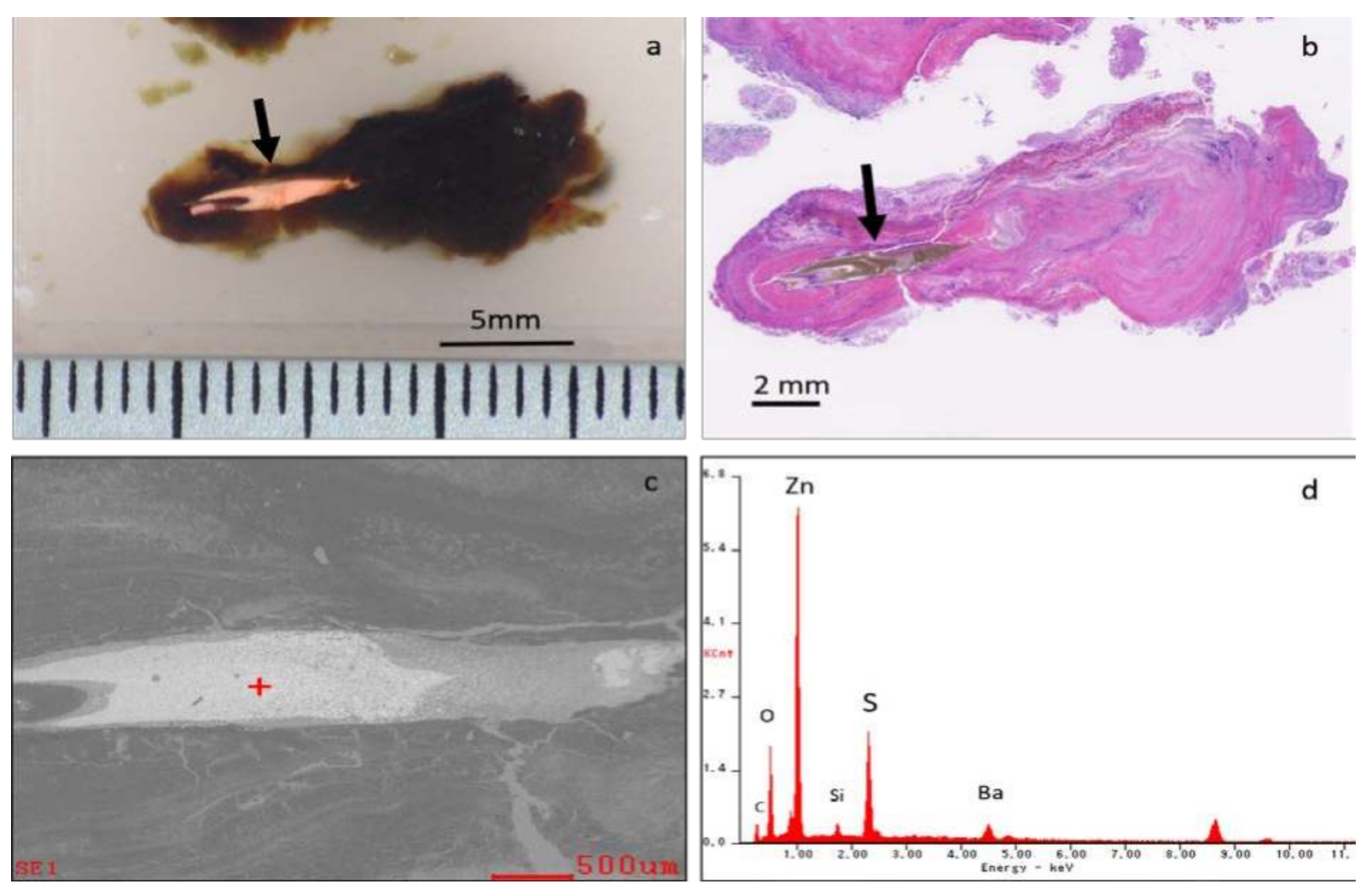

Fig. 2 (a) Gross view of the cut surface of the removed specimen, within the paraffin block. Whitish-red substance (arrow) appears similar to a root-filling material: gutta-percha point. (b) Scanning image of the removed specimen, nearly concordant with the gross view in Fig 2a (hematoxylin and eosin). Needle-shaped brownishblack substance (arrow) is visible within the center of the fungal ball. (c) Scanning electron micrograph showing a bright needleshaped area in the specimen, which corresponds to the whitish-red substance of the paraffin block of 2a and brownish-black material in the histology section of $2 \mathrm{~b}$. The red cross indicates the area where $\mathrm{X}$-ray analysis was performed. (d) An energy-dispersive X-ray analysis of the bright area of $2 \mathrm{c}$ shows peaks of zinc $(\mathrm{Zn})$, sulfur $(\mathrm{S})$, and barium (Ba), as well as peaks of ubiquitous elements.
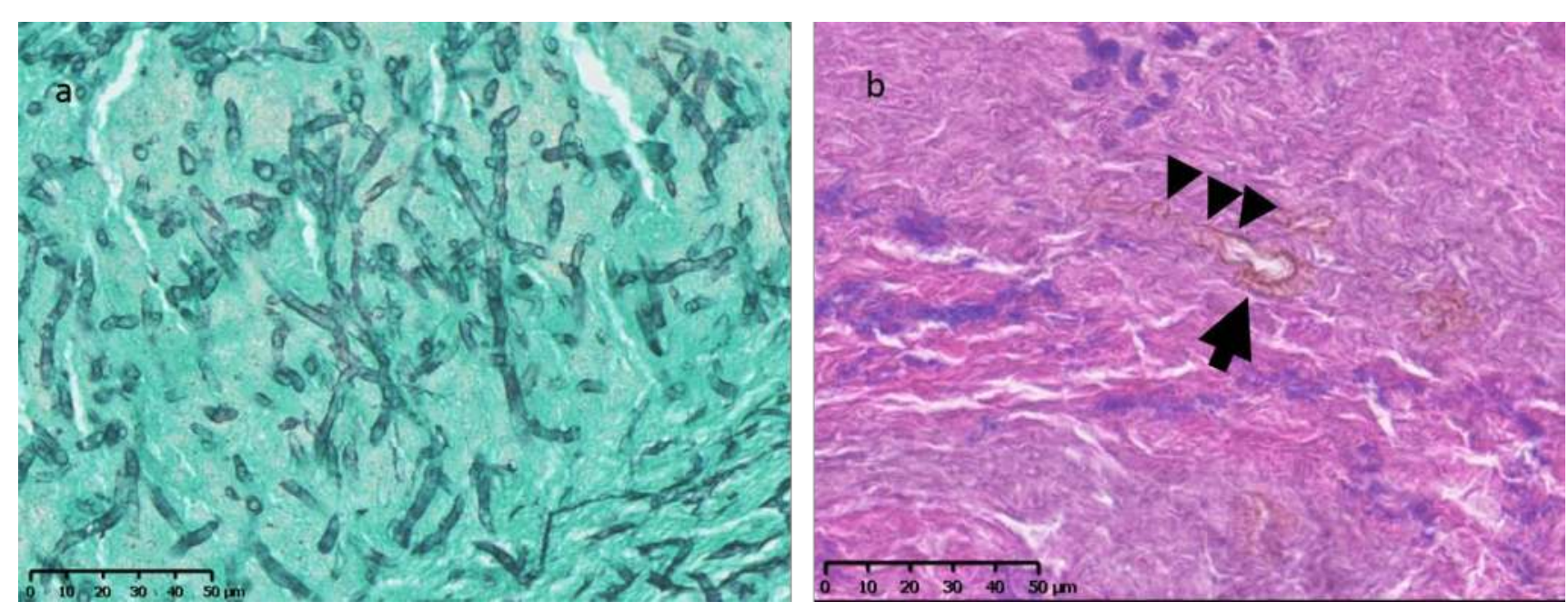

Fig. 3 Enlarged view of the removed specimen of 2b. (a) Dichotomously branching septate hyphae of the hyphal mat (Grocott stain) (b) Conidial heads in the hyphal mat. A brown, round conidial head (arrow) is visible with a continuous wide tube-like conidiophore (arrow heads) (hematoxylin and eosin)
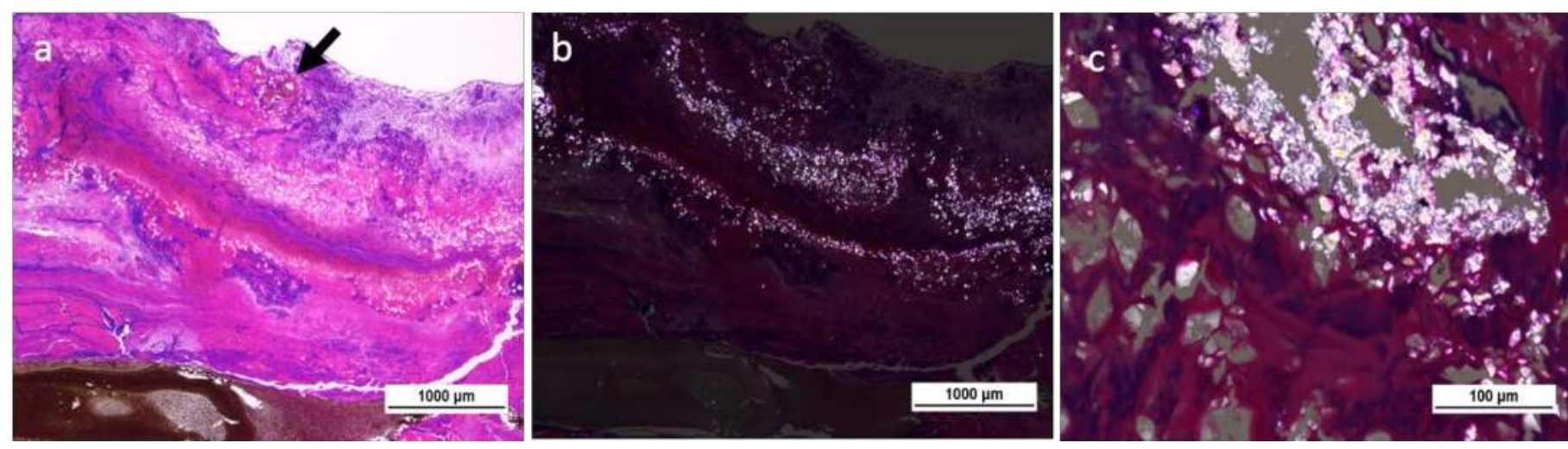

Fig. 4 (a) Enlarged view of a portion of Fig. 2b. Bands of translucent calcium oxalate crystals are present on the hyphal mat. An arrow indicates cleistothecia. Brown root-filling material is visible on the lower half of the picture (hematoxylin and eosin).

(b) The same section as $4 \mathrm{a}$, viewed under polarized light. Bands of birefringent crystals are visible (hematoxylin and eosin under polarized light). (c) Enlarged view of 4b. Shapes of calcium oxalate crystals are rosette, sheaf-like grouping, and rhombus (hematoxylin and eosin under polarized light)
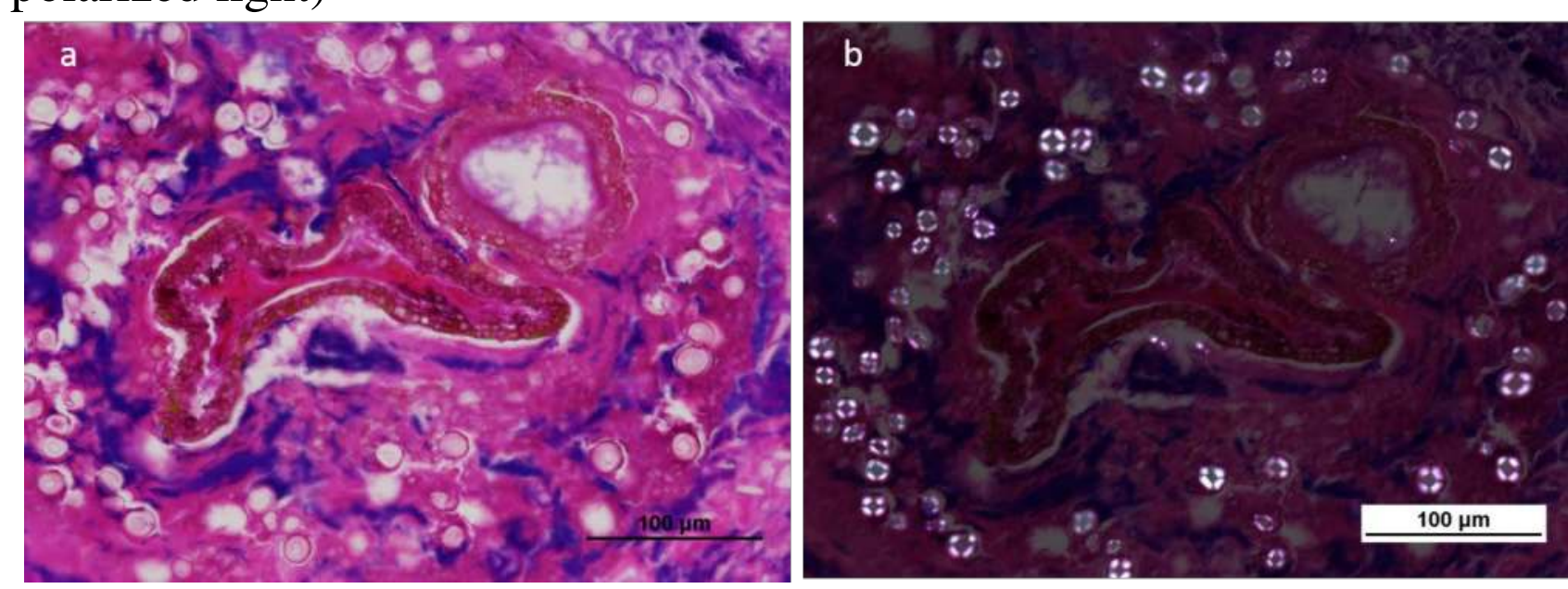

Fig. 5 Enlarged view of two cleistothecia indicated by the arrow in Fig. 4a. (a) One cleistothecium is deformed, whereas the other is round. The wall of the cleistothecia is similar to a brown basket. A few ascospores (arrow) are visible within the cleistothecia. The cleistothecia are surrounded by thick-walled, hyaline, globose Hülle cells (hematoxylin and eosin). (b) The same section as 5a, viewed under polarized light. The Hülle cells show a Maltese-cross pattern of birefringence (hematoxylin and eosin under polarized light) 